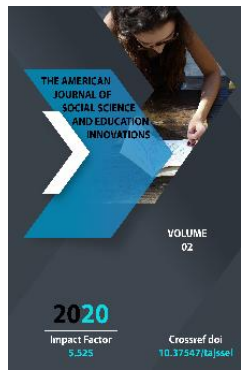

\title{
Method Of Learning New Words
}

\author{
Nematova Shahribonu Gani Qizi \\ Student Of Uzbek State World Languages University, Uzbekistan
}

Copyright: Original content from this work may be used under the terms of the creative commons attributes 4.0 licence.

\section{ABSTRACT}

As a language learner, you work hard to expand your vocabulary. You plough through new words every day, make long lists of words and practise with flashcards. However, when it comes to speaking, the new words seem to fall out of your head, so you resort to your old friends - words you already know and have used many times - again and again. Remembering and using new words in speech is often a challenge for language learners. This article goes on to think about word memorization and its methods.

\section{KEYWORDS}

Reading a lot of books, Write, Look, Cover, Repeat (WLCR), Flashcarding, Work in a Context, Stickies Everywhere, Study Linguistics, use your inner voice, repetition, grouping words

\section{INTRODUCTION}

It is well known that learning words in French is a problem for many people. Most people find it difficult to memorize words in French only by studying grammar in depth, while the following methods help in word memorization.
Vocabulary acts as a strong basis for any language. You cannot be good at any language if you lack proper vocabulary. If you want to learn any language fluently then you need to get a strong grip on vocabulary. Moreover, it is 
the one ingredient that beautifies the language in the right way. Now, it is not so tough as it sounds, but learning vocabulary can be considered as an advanced topic in language learning. Be it any language, target it and learn it as much as possible by following the steps mentioned below.Vocabulary is one of the pillars of language learning and especially significant for the advanced learner. Once you are familiar with the basic structures of your target language, you'll want to start saying and understanding more words! One of my friends recently asked me:

I'd like to get some system for vocab learning in place. Have you any recommendation?

....and I thought this makes an awesome blog topic. So here are my top techniques for learning vocabulary.

\section{MATERIALS AND METHODS}

The 6 Best Techniques for Learning Vocabulary.

\section{Write, Look, Cover, Repeat (WLCR).}

This is the ultimate classic. For me, vocab learning has always been a notebook and pen type exercise. The physical motion of writing something down is very useful as it satisfies the needs of haptic learners. Take a pad, draw a vertical line in the middle and write the word on one side in your native/source language and on the other side in your target language. Memorize the list, then cover one side and tick off all that you remember. Then repeat. Supercharging your WLCR:

- Add another Write step for all the words you couldn't remember first time round, then start again. And repeat. The list of words you can't remember should get smaller every time.
- Satisfy your aural learning style through the version write, mumble, cover, repeat speaking out each target language word every time. Or record them all and listen back to the vocabulary list a few times.

- Colour code your list, for example to highlight different genders or word types.

\section{Get them all.}

This technique is very well suited to learning by immersion. You acquire new words by carrying a dictionary everywhere (anything electric has them on these days) and then making a note of new words wherever you come across them. These form your daily vocabulary list. Learn the words, revisit the source, move on to a related text or video for repetition. Keep going until you remember all (or 80\%, 70\%...it's your choice!) of the words from your list, then find a new source. Your memory willbuild the connection between source and vocab, allowing you to remember the word by wher you learnt it from.

\section{Flashcarding}

Flashcards are great for learners who work very well with visual clues. You can either make sets combining an image with a word in the target language, or just write the words in source and target language. Different colours come in handy here, and many online products also offer to make life easier for you. What makes Flashcarding different from WLCR is that you cannot rely on memorising words by order - they come at you at random and you must be ready for anything. Personally, I perceive this as a hurdle and it's never been my thing. But on the other hand, what better way of preparing for real life?

\section{Work in a Context}


Memory research tells us that building links between data helps us remember things and the way to make sense of that is to put the vocab into use. A classic memory technique would be to connect every word with a strong image (imagine the grumpy ticket clerk huffing and handing you two tickets). Alternatively, come up with a story that includes all your vocabulary.I also like to to work in blocks of different topics, for example you can focus on cookery for a few weeks. That way, the most common words will reoccur and you learn not only the word, but how to use it.

Again, immersion is key here and make sure you produce language in context. Write a full sentence using your new word. Update your Facebook in the target language. Use it 5 times in a conversation. Whatever it takes, just make sure you have satisfied both repetition and context.

\section{Stickies Everywhere}

Sticky notes were made to serve as reminders, so we shall use them for that! Put contextual vocabulary in strategic places, or write yourself little messages in the target language. After a week of "Bonne journée, Bonsoir" on your mirror, it might just sink in.

\section{Study Linguistics}

Many words are made up of parts of other words. So this one requires a bit of study, but it will make your vocabulary learning the smartest it has ever been. Become familiar with prefixes and suffixes, word roots and common sources of target language words. For example, many European languagesare Latinbased and their words change through little add-ons.
The next method is grouping words. This method speeds up the learning of words several times. It is easier to learn words when they have something in common. It can be the same topic, word and its opposites, word and its synonyms, or some other logic. Grouping the words helps to memorize them as you link the words not only to its meaning but to some general category too. So, if you accidentally forget, let's say, what car' is, you still may recall that it relates to transport, and then to guess its meaning. Learning synonyms together helps to get the nuances of meanings.

\section{RESULT AND DISCUSSION}

Moreover, it will be easier to organize the following regular practice for these words. It is difficult to find a context to practice the words, that have nothing in common. People who want to learn a new language, do not know the new words and they don't have any idea as to how to fill the gaps in between. Whenever a person wants to learn a new language, then he should continuously talk to himself about various things like what he likes or what he wants to be..and so on. We remember what is relevant to us. Making lists or index cards with random words is not usually an effective way to remember and use these words later. Word lists and index cards are great for revisiting vocabulary you have already learned, but to make a new word stick in your mind, try linking it with something meaningful to you. You will be more likely to remember a new word if it is used in a context you find interesting or are passionate about.Use your inner voice.Learning is essentially an internal process. To learn a word, you need to get into the world of your inner voice. Try the following: listen to a word/phrase once, now listen to it inside your head, then say it inside your head, then say it aloud. Record yourself saying it and 
listen to the recording. Does it sound the way you heard it with your inner ear? Repetition fixes new words in your memory. However, repeating them a hundred times over the course of one day will not be as effective as repeating them a few times over a period of several days or weeks. Use the new word immediately. Then try to recall it in an hour. Review it shortly before you go to bed. Use it again one day later. Finally, review it in a couple of days after that. Another method is reading a lot of books. If a person gets into the habit of reading, then a person is exposed to new words. Always make it a habit to underline the new words and refer to them in the context section and try to understand their meaning. By doing this, you will remember the words and will be able to make its correct usage. Presents an overview of research at the heart of word learning. His paper discusses the advances that have taken place in infant studies of word learning, with a particular focus on the first year or so of life. For this age group, methodology is crucial and in the last 20 year variants of the head-turn preference methodology have proved invaluable. This technique allows two spoken stimuli differing in source location to be compared, with the experimenter measuring the preferred stimulus on the basis of head orientation (i.e. infants will orient their head towards the source of a more interesting stimulus for a longer period of time). It is generally accepted that the first 12 months of life involve a process of refining and tuning of the phonetic categories that are relevant to the infant's language. Contrasts that are unused in a particular language will tend to be lost, so that by 10-12 months of age only the phonetic categories that are relevant to a particular language remain. This important precursor to word learning has led to the assumption that vocabulary acquisition starts in earnest only in the second year.

\section{CONCLUSION}

In conclusion, in order to memorize words, we need to be able to use more of those wordrelated sentences, or the word itself. Another easy method of memorizing words in French is to memorize words by putting them on the cards.

\section{REFERENCES}

1. https://www.fluentlanguage.co.uk/blog/te chniques-for-learning-vocabulary

2. https://www.ncbi.nlm.nih.gov/pmc/articles /PMC2846319/ 